Received 2017 September 8; Revised 2018 January 8; ACCePted 2018 January 26

Preprint typeset using $\mathrm{IATEX}_{\mathrm{E}}$ style emulateapj v. 12/16/11

\title{
VERY LONG BASELINE ARRAY IMAGING OF TYPE-2 SEYFERTS WITH DOUBLE-PEAKED NARROW EMISSION LINES: SEARCHES FOR SUB-KPC DUAL AGNS AND JET-POWERED OUTFLOWS*
}

\author{
Xin Liv ${ }^{1,2,6}$, T. Joseph W. Lazio ${ }^{3,7}$, Yue Shen ${ }^{1,2,5}$, And Michael A. Strauss ${ }^{4}$ \\ Received 2017 September 8; revised 2018 January 8; accepted 2018 January 26
}

\begin{abstract}
This paper presents Very Long Baseline Array (VLBA) observations of 13 double-peaked [O III] emission-line type-2 active galactic nuclei (AGNs) at redshifts $0.06<z<0.41$ (with a median redshift of $z \sim 0.15$ ) identified in the Sloan Digital Sky Survey. Such double-peaked emission-line objects may result from jets or outflows from the central engine or from a dual AGN. The VLBA provides an angular resolution of $\lesssim 10 \mathrm{pc}$ at the distance of many of these galaxies, sufficient to resolve the radio emission from extremely close dual AGNs and to contribute to understanding the origin of double-peaked [O III] emission lines. Of the 13 galaxies observed at $3.6 \mathrm{~cm}(8.4 \mathrm{GHz})$, we detect six at a $1 \sigma$ sensitivity level of $\sim 0.15 \mathrm{mJy}_{\text {beam }}{ }^{-1}$, two of which show clear jet structures on scales ranging from a few milliarcseconds to tens of milliarcseconds (corresponding to a few pc to tens of pc at a median redshift of 0.15). We suggest that radio-loud, double-peaked emission-line type-2 AGNs may be indicative of jet produced structures, but a larger sample of double-peaked [O III] AGNs with high angular resolution radio observations will be required to confirm this suggestion.
\end{abstract}

Subject headings: black hole physics - galaxies: active - galaxies: interactions - galaxies: nuclei galaxies: Seyfert - radio continuum: galaxies - quasars: general

\section{INTRODUCTION}

\subsection{Significance of Binary Supermassive Black Holes}

It is now well established that most, if not all, large galaxies in the local universe have supermassive black holes (SMBHs; > $10^{8} M_{\odot}$ ) in their nuclei (e.g., Kormendy \& Richstone 1995; Ferrarese \& Ford 2005). Further, the merger of galaxies is considered to be an integral aspect of galaxy assembly and evolution, with large galaxies in the local universe potentially having undergone multiple mergers during the course of their evolution. A robust prediction of the galaxy merging process is that the merger product will contain two SMBHs, which will sink to the center of the product via dynamical friction on time scales of order $10^{8} \mathrm{yr}$. Models of this merger process not only predict the formation of a binary $\mathrm{SMBH}$ (e.g., Begelman et al. 1980;

\footnotetext{
* Based, in part, on observations made with the Very Long Baseline Array, obtained at the Long Baseline Observatory. The Long Baseline Observatory is a facility of the National Science Foundation operated under cooperative agreement by Associated Universities, Inc.

${ }^{1}$ Department of Astronomy, University of Illinois at UrbanaChampaign, Urbana, IL 61801, USA

${ }^{2}$ National Center for Supercomputing Applications, University of Illinois at Urbana-Champaign, 605 East Springfield Avenue, Champaign, IL 61820, USA

3 Jet Propulsion Laboratory, California Institute of Technology, M/S 138-308, 4800 Oak Grove Drive, Pasadena, CA 91109, USA

${ }^{4}$ Department of Astrophysical Sciences, Princeton University,

Peyton Hall, Ivy Lane, Princeton, NJ 08544, USA

5 Alfred P. Sloan Foundation Fellow

${ }^{6}$ Email: xinliuxl@illinois.edu

7 Email: Joseph.Lazio@jpl.nasa.gov

9 We distinguish between binary SMBHs and dual SMBHs or dual active galactic nuclei (AGNs). The former are systems for which their mutual gravitational interactions are dominant, while the latter are galaxies or merger products that contain two SMBHs, but for which the separation between the two SMBHs is so large that their motions are dominated by the gravitational potential of
}

Milosavliević \& Merritt 2001; Yu 2002; Merritt 2013), but also claim success in being able to replicate a variety of other aspects of galaxy or quasar properties, including the $M_{\mathrm{BH}}-\sigma$ relation, the quasar luminosity function, the central brightness of galaxies, and the bending or apparent precession of radio jets (e.g., Kauffmann \& Haehnelt 2000; Volonteri et al. 2003; Wvithe \& Loeb 2003; Liu 2004; Hopkins et al. 2008; Kormendy \& Bender 2009; Shen 2009; Kormendy \& Ho 2013).

The kinematics of the two SMBHs at the center of the merger product begins to be dominated by their mutual gravitational interaction, rather than by the gravitational potential of the host, when their separation is (Begelman et al. 1980; Volonteri et al. 2003)

$$
r_{b}=\frac{G\left(m_{1}+m_{2}\right)}{2 \sigma^{2}} \sim 10 \mathrm{pc} \frac{m_{1}+m_{2}}{10^{8} M_{\odot}}\left(\frac{\sigma}{150 \mathrm{~km} \mathrm{~s}^{-1}}\right)^{-2},
$$

for SMBHs of mass $m_{1}$ and $m_{2}$ located within a merger product with a central velocity dispersion $\sigma$. Further dynamical friction by stars in the central region, and possibly gas interactions, cause the binary to harden. For a time, it was thought that the binary separation would cease to shrink at a separation of order $1 \mathrm{pc}$ as stellar interactions become less effective (the "last parsec problem," Begelman et al. 1980; Quinlan 1996; Milosavliević \& Merritt 2001; Yu 2002). Considerable recent attention has focused on interactions between the SMBH binary and the surrounding population of stars, particularly in light of the fact that the stellar population is likely to have an asymmetric spatial or velocity distribution as a result of the merger process itself (e.g., Yu 2002; Berczik et al. 2006; Maver et al. 2007; Lodato et al. 2009; Sesana 2010; Khan et al. 2011; Preto et al. 2011). While the results are not yet conclusive, it appears plausible that such interactions would

the host. 
cause the binary to continue to harden to sub-milliparsec separations within the age of the universe, at which point its separation will shrink inexorably due to the emission of gravitational waves (GWs). Current or future pulsar timing arrays should be able to detect the GW emission from the ensemble of individual binary SMBHs at frequencies of order $10^{-9} \mathrm{~Hz}$ Hobbs et al. 2010; Arzoumanian et al. 2016; Babak et al. 2016), while the last moments of in-spiral should produce GWs with frequencies of order $10^{-6} \mathrm{~Hz}$, which would be detectable by future space interferometers (Amaro-Seoane et al. 2013; Audley et al. 2017). Determining the cosmological density of galaxies containing a pair of SMBHs therefore provides constraints on the rate at which galaxies undergo mergers and the late stages of the merger process (e.g., Yu et al. 2011; Steinborn et al. 2016), as well as being crucial to predicting the amplitudes and rates of GW signals that pulsar timing arrays and future space interferometers will detect (e.g., Sesana et al. 2017).

\subsection{Observational Evidence for Binary and Dual} Supermassive Black Holes and Uncertainties

Despite the theoretically appealing nature of this scenario, however, direct observational evidence for dual and binary SMBHs remains scarce. To date, only a single potential pc-scale binary SMBH is known 10 B2 0402+379 (Maness et al. 2004; Rodriguez et al. 2006), with a (projected) separation of approximately 7 pc; one candidate sub-pc binary SMBH has just been identified in NGC 7674 from direct imaging (with projected separation of $0.35 \mathrm{pc}$; Kharb et al. 2017) using Very long baseline interferometry (VLBI). The fraction of low-redshift AGN pairs on $\sim 5-100 \mathrm{kpc}$ scales is a few percent (e.g., Liu et al. 2011, 2012), and the fraction of intermediate-redshift binary quasars on tens to hundreds of kpc scales is 0.1\% (e.g., Hennawi et al. 2006, 2010; Myers et al. 2008; Shen et al. 2010). Until recently, on kpc scales, there had been only a handful of unambiguous cases of dual AGNs in which both SMBHs are detected in the radio (e.g., 3C 75, Owen et al. 1985), optical (e.g., LBQS 0103-2753, Junkkarinen et al. 2001), or X-rays (e.g., NGC 6240, Mrk 463, and Mrk 739; Komossa et al. 2003; Bianchi et al. 2008; Koss et al. 2011).

The advent of large and uniform spectroscopic surveys, such as the Sloan Digital Sky Survey (SDSS; York et al. 2000), has enabled the identification of large numbers of galaxies with spectroscopic signatures potentially characteristic of dual AGNs on $\sim \mathrm{kpc}$ and sub-kpc scales (e.g., Wang et al. 2009; Liu et al.]2010b; Smith et al. 2010; Ge et al. 2012; Barrows et al. 2013; Comerford et al. 2013; Lyu \& Liu 2016; Yuan et al. 2016), as well as binary SMBHs on sub-pc scales (e.g.,

\footnotetext{
10 There is one candidate sub-pc binary SMBH in OJ 287, which shows a $\sim 12$ year quasi-periodic light curve, and is interpreted with a binary SMBH+accretion disk model (Sillanpaa et al. 1988; Valtonen et al. 2008, 2016). In addition, there is one candidate reported by[Boroson \& Lauer (2009), which was later suggested to be an unusual disk emitter and not a binary SMBH Chornock et al. 2010). There are $\sim 150$ mili-pc binary SMBH candidates proposed based on quasi-periodic quasar light curves (e.g., Graham et al. 2015a b; Liu et al. 2015; D'Orazio et al. 2015, 2016; Charisi et al. 2016; Zheng et al. 2016), although the quasi-periodicity could also be due to single $\mathrm{BH}$ accretion disk instability and/or radio jet precession (e.g., Kudryavtseva et al. 2011) or false periodicities caused by stochastic variability (e.g., Vaughan et al.|2016).
}

Tsalmantza et al. 2011; Eracleous et al. 2012; Ju et al. 2013; Shen et al. 2013; Liu et al. 2014; Runnoe et al. 2015, 2017; Wang et al. 2017). In particular, one such signature is having two spectral-line components associated with AGNs, such as [O III], and with a velocity separation of a few hundred $\mathrm{km} \mathrm{s}^{-1}$ which signals orbital motion on galactic scales (e.g., Zhou et al. 2004; Gerke et al.|2007; Comerford et al.|2009; Xu \& Komossa 2009; Barrows et al. 2012), analogous to a double-lined spectroscopic star binary.

Higher angular resolution follow-up observations of candidates from systematic surveys have dramatically increased the number of dual AGNs on kpc-scale separations(e.g., Liu et al. 2010a, 2017; Fu et al. 2011, 2012; McGurk et al. 2011; Shen et al. 2011; Comerford et al. 2012). However, there are considerable ambiguities associated with identifying dual SMBHs from spectral-line observations alone. Outflows associated with jets and rotating disks may also produce double-peaked narrow emission lines in AGNs (Axon et al. 1998; Xu \& Komossa 2009; Crenshaw et al. 2010; Rosario et al. 2010; Smith et al. 2010, 2011; Comerford et al. 2011; Fischer et al. 2011; Shen et al. 2011), and recent work has suggested that the majority ( $\gtrsim 50 \%$ ) of double-peaked narrow emission-line AGNs are likely due to complex narrow-line kinematics around single AGNs (Shen et al. 2011; Fu et al. 2012; Nevin et al. 2016).

\subsection{This Work: High-resolution Imaging with VLBA}

Considering the sizes of the narrow-line regions (NLRs) responsible for the [O III] lines and the evolutionary stages of a dual SMBH, the physical separation between dual AGNs with two distinct NLRs can be as small as $\sim 30$ pc (e.g., Schmitt et al. 2003; Greene et al. 2011, depending on AGN luminosity), corresponding to an angular separation of order 10 mas at a typical redshift $z \sim 0.1$; actual separations could be smaller when projected on the sky. Dual AGNs with such small separations would show double-peaked narrow emission lines given typical orbital velocities of the individual NLRs being a few hundred $\mathrm{km} \mathrm{s}^{-1}$ and typical velocity dispersions of $\lesssim$ a few hundred $\mathrm{km} \mathrm{s}^{-1}$ of the NLR gas clouds, provided that the two NLRs are not yet fully merged. Only with a full assessment of all the candidate dual AGNs at various separations will we be able to put robust constraints on aspects merger scenarios, such as the merger fraction, the dynamics of merging SMBH pairs, the significance of mergers in triggering AGNs, and the separations of the merging components at which AGNs are triggered.

VLBI techniques routinely produce images with milliarcsecond resolutions, equivalent to a linear separation of order $10 \mathrm{pc}$ (at $z \sim 0.1$ ), which is far higher than can be obtained at other wavelengths even with adaptive optics in the optical/near-infrared. Further, VLBI observations have traditionally been sensitive to high brightness temperature structures in the inner regions and nuclei of galaxies, such as AGNs and jets. Thus, the combination of spectroscopic surveys and VLBI imaging offers a powerful means of searching for sub-kpc dual SMBHs that is less biased due to spatial resolution limit compared with other observations. VLBI will also be sensitive to radio jets of scales of tens of parsecs; the gas outflows they 
drive may be responsible for the double-peaked narrow emission lines in AGNs. In essence, VLBI observations can probe a new spatial regime in the parameter space of dual SMBHs or jets.

This paper presents Very Long Baseline Array (VLBA; Napier et al. 1994) observations of a subset of the double-peaked narrow emission-line AGNs identified by Liu et al. (2010b) from the SDSS DR7 (Abazajian et al. 2009). The objective was to assess the radio detection rate and the fraction of objects that have compact and binary radio components in their cores. In $\$ 2$, we summarize our VLBA observations; in 43 , we discuss the six galaxies that we detect radio emission from; and in 4 , we discuss what our results imply about sub-kpc dual SMBHs and implications for future observations. Throughout, we assume a cosmology of a Hubble constant of $70 \mathrm{~km} \mathrm{~s}^{-1} \mathrm{Mpc}^{-1}$, a matter density $\Omega_{m}=0.3$, and a vacuum energy density of $\Omega_{\mathrm{vac}}=0.7$.

\section{TARGET SELECTION, OBSERVATIONS, AND DATA REDUCTION AND ANALYSIS}

As an initial search for close pairs (both physical and in projection) and to demonstrate the feasibility of a larger effort, we observed 13 type-2 Seyfert AGNs with double-peaked [O III] $\lambda \lambda 4959,5007$ emission lines with VLBA (Program BL170, PI: Liu). The observations were at $3.6 \mathrm{~cm}(8.4 \mathrm{GHz})$ with a total bandwidth of $32 \mathrm{MHz}$. This observational wavelength was motivated by two factors: (1) the angular resolution of the VLBA at $3.6 \mathrm{~cm}$ is approximately 1 mas, more than sufficient to detect any sub-kpc dual AGNs given their expected separations (\$1) and (2) the VLBA is at its most sensitive at this wavelength.

\subsection{Target Selection}

Our target sample was drawn from the 167 doublepeaked emission-line AGNs identified by Liu et al. (2010b). We first selected galaxies either having counterparts in the Faint Images of the Radio Sky at Twenty Centimeters survey (FIRST; Becker et al. 1995) or that had existing observations within the VLA Archive from which the radio structure could be assessed. Of all the radio-bright objects in our parent sample (77 in 167 objects being detected by FIRST), we selected 12 objects that have $1.4 \mathrm{GHz}$ flux densities above $7 \mathrm{mJy}$ (from among a total of 23), and which appear unresolved in the core to FIRST (angular resolution $\sim 5 "$ ). The VLA Archival observations, where they exist, were used to confirm that the sources remain compact on sub-arcsec scales, to estimate their flux densities at higher frequencies to ensure adequate signal-to-noise ratios $(\mathrm{S} / \mathrm{N})$ for imaging, and improve the positional information for the new VLBA observations. We supplemented the sample with one additional object (SDSS J135251.22+654113.2) from the Liu et al. (2010b) sample that was not covered by the FIRST survey, but which had existing radio observations within the VLA Archive and satisfied our selection criteria. The median redshift of our target sample is $\sim 0.15$. Figure 1 shows the $[\mathrm{O}$ III] emission-line flux

\footnotetext{
${ }^{11}$ Had we have observed sources that were resolved by FIRST and not detected anything, one possibility would be that there is no milliarcsecond (mas) structure. Our preference toward unresolved FIRST sources is largely to assure a likely high detection rate.
}

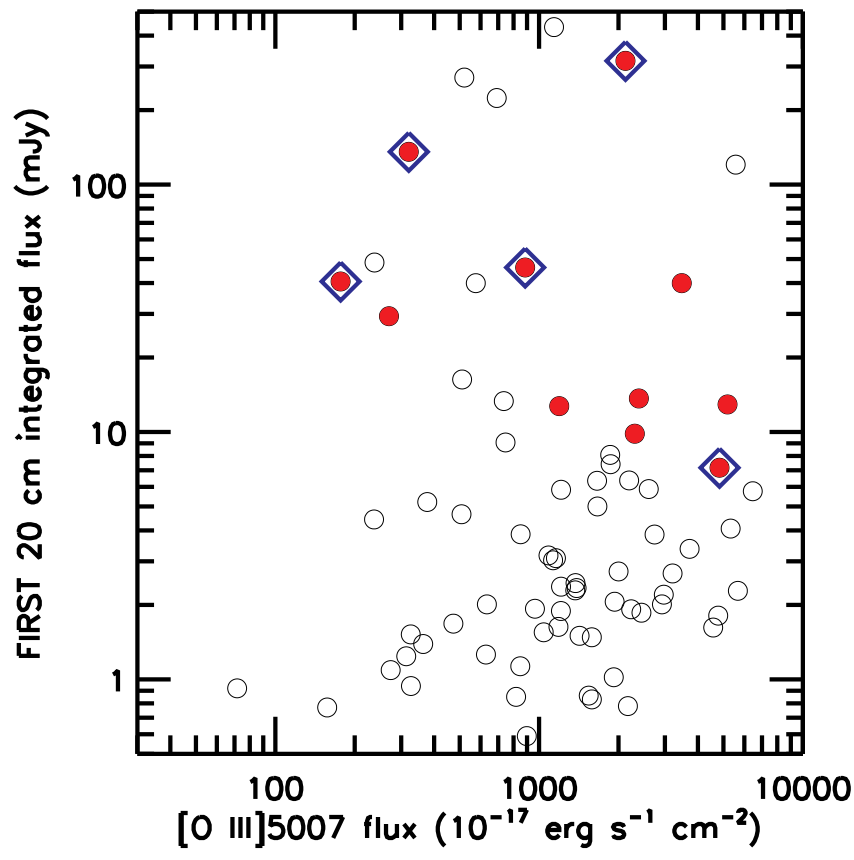

FIG. 1.- [O III $]$ emission-line flux versus FIRST integrated flux at $20 \mathrm{~cm}$ for our VLBA targets (red filled circles). Also shown for comparison are the other FIRST-detected sources (open circles) in the parent sample of double-peaked, narrow-line AGNs from Liu et al. (2010b). Typical measurement uncertainties are $\sim 2.6 \times$ $10^{-16} \mathrm{erg} \mathrm{s}^{-1} \mathrm{~cm}^{-2}$ for [O $\left.\mathrm{III}\right]$ emission-line flux and $\sim 0.15 \mathrm{mJy}$ for FIRST integrated flux at $20 \mathrm{~cm}$. Targets that were detected by our VLBA observations are marked with blue open diamonds. The VLBA-detected target SDSS J135251.22+654113.2 is not shown here, because it was not covered by the FIRST survey.

versus FIRST integrated flux at $20 \mathrm{~cm}$ for our targets as compared with the other radio-bright objects in our parent sample. Our VLBA targets are selected to have high radio fluxes at $20 \mathrm{~cm}$, but their [O III] emission-line fluxes sample the full range of the parent sample.

\subsection{VLBA Observations}

The VLBA observations were conducted in four sessions, with each session lasting approximately $6 \mathrm{hr}$, in the interval between 2010 March 30 and June 5. Target flux densities at $8.4 \mathrm{GHz}$ ranged from 135 to $1.5 \mathrm{mJy}$, with a median flux density of $40 \mathrm{mJy}$. Anticipating that some fraction of the flux density measured by FIRST might be resolved out by the VLBA observations, we used phase referencing for all of the observations, cycling between the target source ( 3 minutes) and a phase reference calibrator (2 minutes). Typical on-source integration times were 40 minutes, implying expected thermal noise levels of approximately $0.15 \mathrm{mJy}^{\text {beam }}{ }^{-1}$. The phase reference calibrator was selected from the VLBA calibrator database. In addition, each session included short scans of a fringe finding calibrator (either 4C 39.25 or $3 \mathrm{C} 454.3)$ and a source for checking the amplitude response (J1310+3220, DA 193, or OJ 287).

\subsection{Data Reduction and Analysis}

We adopted standard data reduction procedures using the Astronomical Image Processing System 12$]$ (version 31DEC10). Specifically, we performed amplitude

\footnotetext{
12 http://www.aips.nrao.edu/index.shtml
} 
TABLE 1

VLBA 3.6 Cm (8.4 GHz) Detected Galaxies

\begin{tabular}{|c|c|c|c|c|c|c|c|c|}
\hline $\begin{array}{c}\text { SDSS Name } \\
\text { (1) }\end{array}$ & $\begin{array}{l}\text { Redshift } \\
\text { (2) }\end{array}$ & $\begin{array}{c}S_{\text {FIRST }} \\
(\mathrm{mJy}) \\
(3)\end{array}$ & $\begin{array}{c}\sigma_{\mathrm{FIRST}} \\
\left(\text { mJy beam }^{-1}\right) \\
(4)\end{array}$ & $\begin{array}{c}\theta_{\mathrm{maj}} \times \theta_{\min } \\
(\mathrm{mas} \times \text { mas }) \\
(5)\end{array}$ & $\begin{array}{c}\sigma_{I} \\
\left(\mathrm{mJy} \mathrm{beam}^{-1}\right) \\
(6)\end{array}$ & $\begin{array}{c}I \\
\left(\mathrm{mJy} \mathrm{beam}^{-1}\right) \\
(7)\end{array}$ & $\begin{array}{c}S \\
(\mathrm{mJy}) \\
(8)\end{array}$ & $\begin{array}{c}L_{\nu} \\
\left(10^{23} \underset{\mathrm{W}}{\mathrm{W}} \mathrm{Hz}^{-1}\right) \\
(9)\end{array}$ \\
\hline J091201.68+532036.6 & 0.1017 & 135.65 & 0.16 & $1.92 \times 1.07$ & 0.15 & 2.58 & 43.0 & 8.3 \\
\hline J124358.36-005845.4 & 0.4092 & 40.58 & 0.15 & $1.94 \times 0.87$ & 0.16 & 21.87 & 23.27 & 130 \\
\hline $\mathrm{J} 135251.22+654113.2$ & 0.2064 & $\mathrm{~N} / \mathrm{A}$ & $\mathrm{N} / \mathrm{A}$ & $1.56 \times 0.78$ & 0.14 & 21.84 & 28.48 & 33 \\
\hline $\mathrm{J} 231051.95-090011.9$ & 0.0733 & 46.22 & 0.15 & $2.35 \times 0.90$ & 0.18 & 3.15 & 3.95 & 0.48 \\
\hline $\mathrm{J} 233313.17+004911.8$ & 0.1699 & 316.33 & 0.10 & $2.16 \times 0.94$ & 0.24 & 4.56 & 5.22 & 3.9 \\
\hline
\end{tabular}

Note. - Column (1): SDSS designation with J2000 coordinates. Column (2): SDSS spectroscopic redshift. Column (3): FIRST integrated flux density at $20 \mathrm{~cm}$; N/A means source is not covered by the FIRST survey. Column (4): RMS noise at $20 \mathrm{~cm}$ in the FIRST survey map; N/A means source is not covered by the FIRST survey. Column (5): major and minor axis of the CLEAN restoring beam. Column (6): 1- $\sigma$ noise level determined within a region $2^{\prime \prime}$ square centered on the source. Column (7): peak brightness. Column (8): peak flux density. Column (9): implied radio luminosity density.

TABLE 2

VLBA 3.6 CM (8.4 GHz) Undetected Galaxies

\begin{tabular}{|c|c|c|c|c|c|c|}
\hline $\begin{array}{l}\text { SDSS Name } \\
(1)\end{array}$ & $\begin{array}{l}\text { Redshift } \\
\quad(2)\end{array}$ & $\begin{array}{c}S_{\text {FIRST }} \\
(\mathrm{mJy}) \\
(3)\end{array}$ & $\begin{array}{c}\sigma_{\mathrm{FIRST}} \\
\left(\mathrm{mJy} \mathrm{beam}^{-1}\right) \\
(4)\end{array}$ & $\begin{array}{c}\theta_{\mathrm{maj}} \times \theta_{\mathrm{min}} \\
(\mathrm{mas} \times \mathrm{mas}) \\
(5)\end{array}$ & $\begin{array}{c}\sigma_{I} \\
\left(\mathrm{mJy} \text { beam }^{-1}\right) \\
(6)\end{array}$ & $\begin{array}{c}L_{\nu} \\
\left(10^{21} \mathrm{~W} \mathrm{~Hz}^{-1}\right) \\
(7)\end{array}$ \\
\hline J000911.58-003654.7 & 0.0733 & 39.95 & 0.15 & $1.64 \times 0.73$ & 0.21 & $<7.7$ \\
\hline J073849.75+315611.9 & 0.2973 & 29.37 & 0.15 & $1.54 \times 0.63$ & 0.18 & $<140$ \\
\hline J080337.32+392633.1 & 0.0655 & 12.92 & 0.14 & $1.53 \times 0.63$ & 0.17 & $<4.9$ \\
\hline J085841.76+104122.1 & 0.1480 & 12.72 & 0.13 & $1.67 \times 0.69$ & 0.17 & $<28$ \\
\hline $\mathrm{J} 110851.04+065901.4$ & 0.1816 & 9.84 & 0.13 & $1.70 \times 0.67$ & 0.21 & $<54$ \\
\hline $\mathrm{J} 135646.11+102609.1$ & 0.1231 & 59.58 & 0.13 & $1.54 \times 0.70$ & 0.20 & $<22$ \\
\hline $\mathrm{J} 171544.05+600835.7$ & 0.1569 & 13.66 & 0.14 & $1.55 \times 0.79$ & 0.19 & $<36$ \\
\hline
\end{tabular}

Note. - Column (1): SDSS designation with J2000 coordinates. Column (2): SDSS spectroscopic redshift. Column (3): FIRST integrated flux density at $20 \mathrm{~cm}$. Column (4): RMS noise at $20 \mathrm{~cm}$ in the FIRST survey map. Column (5): major and minor axis of the CLEAN restoring beam. Column (6): 1- $\sigma$ noise level determined within a region $2^{\prime \prime}$ square centered on the source location. Column (7): $3 \sigma$ upper limit on the radio luminosity density, at the galaxy's redshift.

calibration of the visibility data using a priori knowledge of the system temperature information for the antennas, applied a parallactic correction for the rotation of the antenna feed orientation during the observation, determined the spectral bandpass response, and calculated residual delays and fringe rates (fringe fitting). A bandpass response function was determined because, even though the observations are intended to be continuum, the data were acquired in a spectral mode. These calibration steps were performed on the calibrators, most notably on the phase reference calibrators, and then interpolated onto the target sources. The full VLBA was used for these observations. Notionally, the array has 10 antennas, although because of the editing of data, the number of antennas being used in a given scan varied between nine and 10 .

Each target source was then imaged. In all cases, a region approximately $2^{\prime \prime} \times 2^{\prime \prime}$ was searched, corresponding to a linear distance of approximately $5 \mathrm{kpc}$ at the median redshift of 0.15 . Typical resolutions obtained were approximately 1.1 mas, corresponding to an equivalent linear distance of $2.7 \mathrm{pc}$, again at the median redshift. Tables 1 and 2 summarize the characteristics of the images, and derived quantities from the images, for the detected and undetected galaxies, respectively. For all galaxies, we report the SDSS spectroscopic redshift $z$; the major and minor axis of the CLEAN restoring beam, $\theta_{\text {maj }}$ and $\theta_{\text {min }}$; and the noise level in the image $\sigma_{I}$. For the detected galaxies, we report the peak brightness $I$ and the integrated flux density $S$. We also report the implied (spectral) luminosity $L_{\nu}$ at the redshift of the detected galaxies and the $(3 \sigma)$ upper limit on $L_{\nu}$ based on the image noise level for the undetected galaxies. For the sources that were detected, all were found within 0. ".05 of their nominal position (determined either from optical images or from lower-resolution radio observations). For the sources that were not detected, the stated image noise levels were determined within the searched region.

\section{INDIVIDUAL GALAXIES}

In this section, we present the images of the detected galaxies and discuss our results in the context of other observations of these galaxies in the literature. The images we present are much smaller than the full $2^{\prime \prime} \times 2^{\prime \prime}$ region initially searched for emission as, in all cases, we found the emission to be not only compact but relatively close to the center of the region searched.

\subsection{SDSS J091201.68+532036.6}

The $z=0.1017$ AGN contains double-peaked [O III] $\lambda \lambda 4959,5007$ lines in its SDSS fiber spectrum (Figure 2), with peaks blueshifted and redshifted from the systemic velocity by $193 \mathrm{~km} \mathrm{~s}^{-1}$ and $208 \mathrm{~km} \mathrm{~s}^{-1}$, respectively (Liu et al. 2010b). SDSS images show that the host galaxy has a close companion to the southwest 

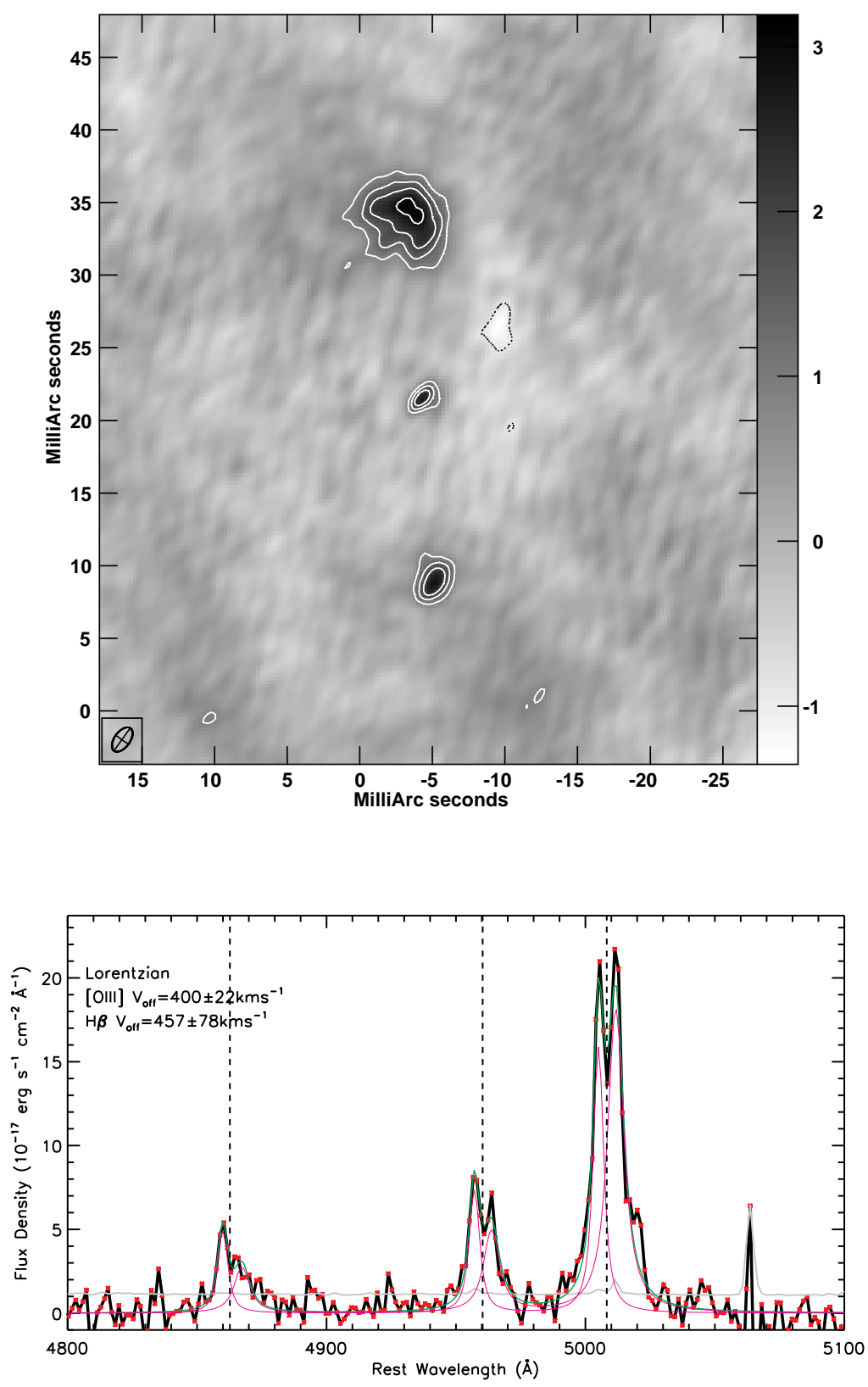

FIG. 2.- Top: VLBA $8.4 \mathrm{GHz}$ image of SDSS J091201.68+532036.6. The gray scale is linear over the range -1.34 to $3.18 \mathrm{mJy}$ beam ${ }^{-1}$. The beam is 1.92 mas $\times 1.07$ mas (corresponding to $3.6 \mathrm{pc} \times 2.0 \mathrm{pc}$ at the redshift of the galaxy $z=0.1017$ ), and the noise level is 0.15 mJy beam ${ }^{-1}$. Contours are given with the levels set to be $-3,3,5,7$, and 10 times the noise level in the image. Bottom: SDSS spectrum (flux density shown in red points connected with black curves and $1 \sigma$ error shown in gray; subtracted for host-galaxy stellar continuum) along with our best fits (model in green and individual velocity components in magenta) for the $\mathrm{H} \beta$ - $[\mathrm{O}$ III $]$ region. The vertical lines are drawn at the systemic redshift from host-galaxy stellar absorption. Labeled on the plot are our best-fit model function (Lorentzian or Gaussian) and the velocity offsets between the double-peaked components measured for [O III] $\lambda \lambda 4959,5007$ and for $\mathrm{H} \beta$.

at a projected separation of $3.7 \mathrm{kpc}\left(2 .^{\prime \prime} 0\right)$. It is unclear whether the SDSS fiber spectrum is significantly contaminated by light from the companion. We have a spectrum of the companion from MMT, but it shows no emission lines and does not have the $\mathrm{S} / \mathrm{N}$ to measure a redshift. Its SDSS photometric redshift (Ovaizu et al. 2008) is consistent with the redshift of the AGN.

This galaxy contains a radio source that has been detected in surveys over the frequency range $0.365-$
8.4 GHz (Becker et al. 1991; Gregorv \& Condon 1991; Douglas et al. 1996; Condon et al. 1998). In addition, Chandra X-ray observations show a point source with a flux of $6.8_{-1.4}^{+1.5} \times 10^{-15} \mathrm{erg} \mathrm{s}^{-1} \mathrm{~cm}^{-2}(0.5-7 \mathrm{keV}$, Evans et al. 2010). In images from both the FIRST survey and the Cosmic Lens All-Sky Survey (CLASS, Mvers et al. 2003), the radio source appears unresolved. There is another, also unresolved, radio source approximately $30^{\prime \prime}$ to the southeast, but it is unclear whether 

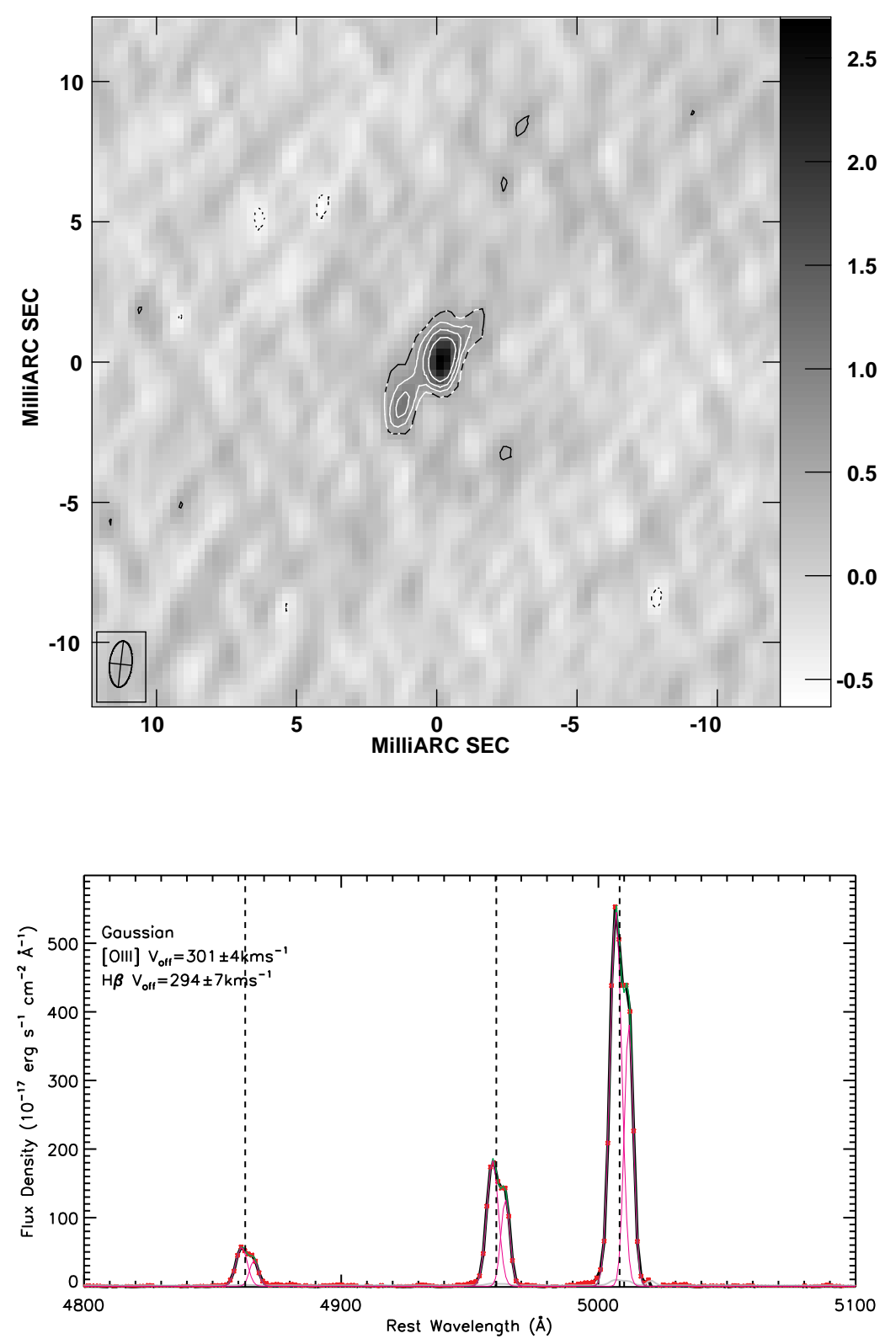

Fig. 3.- Top: VLBA $8.4 \mathrm{GHz}$ image of SDSS J113721.36+612001.2. The gray scale is linear over the range -0.61 to $2.67 \mathrm{mJy}$ beam ${ }^{-1}$. The beam is 1.70 mas $\times 0.80$ mas (corresponding to $3.4 \mathrm{pc} \times 1.6 \mathrm{pc}$ at the redshift of the galaxy $z=0.1112$ ), and the noise level is 0.15 mJy beam ${ }^{-1}$. Contours are given with the levels set to be $-3,3,5,7$, and 10 times the noise level in the image. Bottom: SDSS spectrum (subtracted for host-galaxy stellar continuum) along with our best spectral fits for the $\mathrm{H} \beta$ - $[\mathrm{O}$ III $]$ region. Figure captions and symbols are the same as those in the bottom panel of Figure 2

the two are related.

Figure 2 shows the image resulting from our VLBA observations. The radio source has three components, approximately aligned north-south. The central component is unresolved, the northern component is clearly diffuse and extended, and the southern component is marginally resolved. The flux density obtained by CLASS is approximately $70 \mathrm{mJy}$ whereas our VLBA observations recover approximately $43 \mathrm{mJy}$, indicating that there is (substantial) structure on sub-arcsecond scales that is resolved out by the VLBA observations.

In contrast to the other sources in this sample (Fig- ures 3 7, below), SDSS J091201.68+532036.6 shows substantial sub-arcsecond-scale structure, though it also has a compact core component, with a flux density of $3 \mathrm{mJy}$, comparable to the flux densities measured for compact components of other sources in our sample. While this source is unusual in our sample (and that of Tingay \& Wayth 2011), it is not unprecedented. MultiElement Radio-Linked Interferometer Network (MERLIN) observations of sources commonly show structure on sub-arcsecond scales (e.g., Filho et al. 2006; Williams et al. 2017), most notably including the doublepeaked emission-line source 3C 316 (An et al. 2013). 
Moreover, we note two potential selection effects. First, while the extended emission contributes significantly to the total flux density of the source, its typical surface brightness is a factor of 2-3 lower than that of the compact component or the peak brightnesses in the north and south components. Modest changes in the observations (e.g., had we observed for only 20 minutes instead of 40 minutes) would have resulted in less of the extended emission being apparent. Second, typical observations of lower luminosity radio sources have sought to address whether there is a compact core component. With such a focus, it is possible that diffuse emission located tens of milliarcseconds from a core component would not have been noticed. Indeed, a limited sampling of the literature shows a number of VLBI images of lower luminosity radio sources for which the typical image is 10 mas $\times 10$ mas (cf. Figure 2).

\subsection{SDSS J110851.04+065901.4}

This $z=0.1816$ AGN contains double-peaked [O III] $\lambda \lambda 4959,5007$ lines in its SDSS fiber spectrum, with peaks blueshifted and redshifted from the systemic velocity by $95 \mathrm{~km} \mathrm{~s}^{-1}$ and $114 \mathrm{~km} \mathrm{~s}^{-1}$, respectively (Liu et al. 2010b). It represents our only target that hosts a kpcscale dual AGN detected by Chandra in the X-rays (Liu et al. 2013).

Liu et al. (2010a) resolved a dual AGN in this galaxy using near-IR imaging and optical slit spectroscopy, with an approximate separation of $00^{\prime \prime} 7$, corresponding to a linear separation of $2.1 \mathrm{kpc}$ at its redshift (Liu et al. 2013). Both ground-based imaging in the NIR (Liu et al. 2010a; Fu et al. 2012) and HST Y-band imaging (Liu et al. 2013) show disturbance in the galaxy surface brightness profile, suggesting tidal interactions. Liu et al. (2013) confirmed its dual AGN nature in the X-rays based on Chandra observations combined with constraints on X-ray contribution from star formation estimated from HST $U$-band imaging. We do not detect a radio source, at a $5 \sigma$ limit of 1.1 mJy beam $^{-1}$.

\subsection{SDSS J113721.36+612001.2 (4C 61.23)}

This $z=0.1112$ AGN contains double-peaked [O III] $\lambda \lambda 4959,5007$ lines in its SDSS fiber spectrum (Figure 31), with peaks blueshifted and redshifted from the systemic velocity by $87 \mathrm{~km} \mathrm{~s}^{-1}$ and $214 \mathrm{~km} \mathrm{~s}^{-1}$, respectively (Liu et al. 2010b). The SDSS images show no evidence for tidal disturbance, which is also confirmed by an HST $Y$-band image (in preparation). This galaxy contains a radio source that has been detected in surveys at least over the frequency range $38 \mathrm{MHz}$ to $5 \mathrm{GHz}$ Gower et al. 1967; Becker et al. 1991; Gregory \& Condon 1991; White \& Becker 1992; Hales et al. 1995; Douglas et al. 1996; Cohen et al. 2007). Lara et al. (2001) classify it as a Faranoff-Riley II radio galaxy. Their VLA image (at $5 \mathrm{GHz}$ ) shows it to be symmetric, with the lobes oriented at $135^{\circ} \mathrm{E}$ from $\mathrm{N}$.

Figure 3 shows our VLBA image. Although superficially an apparent double, with the second component approximately 2 mas to the southeast of the primary component, the orientation of the milliarcsecond structure is similar to that of the arcsecond FR II structure, suggesting that the small-scale fainter component could be a portion of the jet that is feeding the southeast largescale radio lobe.

\subsection{SDSS J124358.36-005845.4}

This $z=0.4092$ AGN shows double-peaked [O III] $\lambda \lambda 4959,5007$ lines in its SDSS fiber spectrum (Figure 4), with peaks blueshifted and redshifted from the systemic velocity by $360 \mathrm{~km} \mathrm{~s}^{-1}$ and $161 \mathrm{~km} \mathrm{~s}^{-1}$, respectively (Liu et al. 2010b). The SDSS images show no evidence for tidal disturbance, although both the image quality and sensitivity may be too low to put a strong constraint. This galaxy contains a radio source that has been detected in surveys over the frequency range 1.4-5 $\mathrm{GHz}$ (Griffith et al. 1995; Condon et al. 1998), and the radio source appears unresolved in FIRST. Figure 4 shows that the radio source has a compact, unresolved component in our VLBA image. The flux density on these scales is approximately $23 \mathrm{mJy}$ at $3.6 \mathrm{~cm}$, whereas the FIRST flux density is approximately $40 \mathrm{mJy}$ (at 20 $\mathrm{cm})$. The spectrum of the radio source is approximately flat, suggesting that nearly $50 \%$ of the flux density is not recovered by the VLBA image.

Within a region of size $\pm 1^{\prime \prime}$, there are no other radio sources stronger than $1.15 \mathrm{mJy}(7.5 \sigma)$. The nearly equatorial declination and limited hour angle coverage contribute to a point spread function (beam) with high secondary peaks, such that a more stringent limit cannot be placed.

\subsection{SDSS J135251.22+654113.2}

This $z=0.2064$ AGN shows double-peaked [O III] $\lambda \lambda 4959,5007$ lines in its SDSS fiber spectrum (Figure 5), with peaks blueshifted and redshifted from the systemic velocity by $108 \mathrm{~km} \mathrm{~s}^{-1}$ and $265 \mathrm{~km} \mathrm{~s}^{-1}$, respectively (Liu et al. 2010b). The SDSS images show tentative evidence for tidal disturbance, possibly related to a companion to the southeast of the main galaxy.

This galaxy contains a radio source which has been detected in surveys at least over the frequency range $74 \mathrm{MHz}$ to $8 \mathrm{GHz}$ (Hales et al. 1990; Becker et al. 1991; Gregory \& Condon 1991; White \& Becker 1992; Douglas et al. 1996; Condon et al. 1998; Cohen et al. 2007). The radio source is unresolved in the CLASS image, with a flux density of $79 \mathrm{mJy}$.

Figure 5 shows our VLBA image. There is at least one faint component located approximately 2.4 mas to the east of the primary component; there may also be a fainter component farther to the east. Our VLBA observations recover approximately $30 \mathrm{mJy}$, indicating that there is likely to be substantial structure on subarcsecond scales.

Within a region of size $\pm 1^{\prime \prime}$, there are no other apparent radio sources brighter than $0.79 \mathrm{mJy}^{\text {beam }}{ }^{-1}$ $(6.6 \sigma)$. Strictly, this is brighter than the nominal statistical threshold, but the brightest pixels in the residual image appear near the edges of the image and there are (negative) pixels with comparable absolute brightnesses.

\subsection{SDSS J231051.95-090011.9}

This $z=0.0944$ AGN shows double-peaked [O III] $\lambda \lambda 4959,5007$ lines in its SDSS fiber spectrum (Figure 6), with peaks blueshifted and redshifted from the systemic velocity by $121 \mathrm{~km} \mathrm{~s}^{-1}$ and $206 \mathrm{~km} \mathrm{~s}^{-1}$, respectively (Liu et al. 2010b). The SDSS images show no evidence for a double stellar core, which is further confirmed by $K_{s}$-band imaging with Magellan/PANIC (Shen et al. 

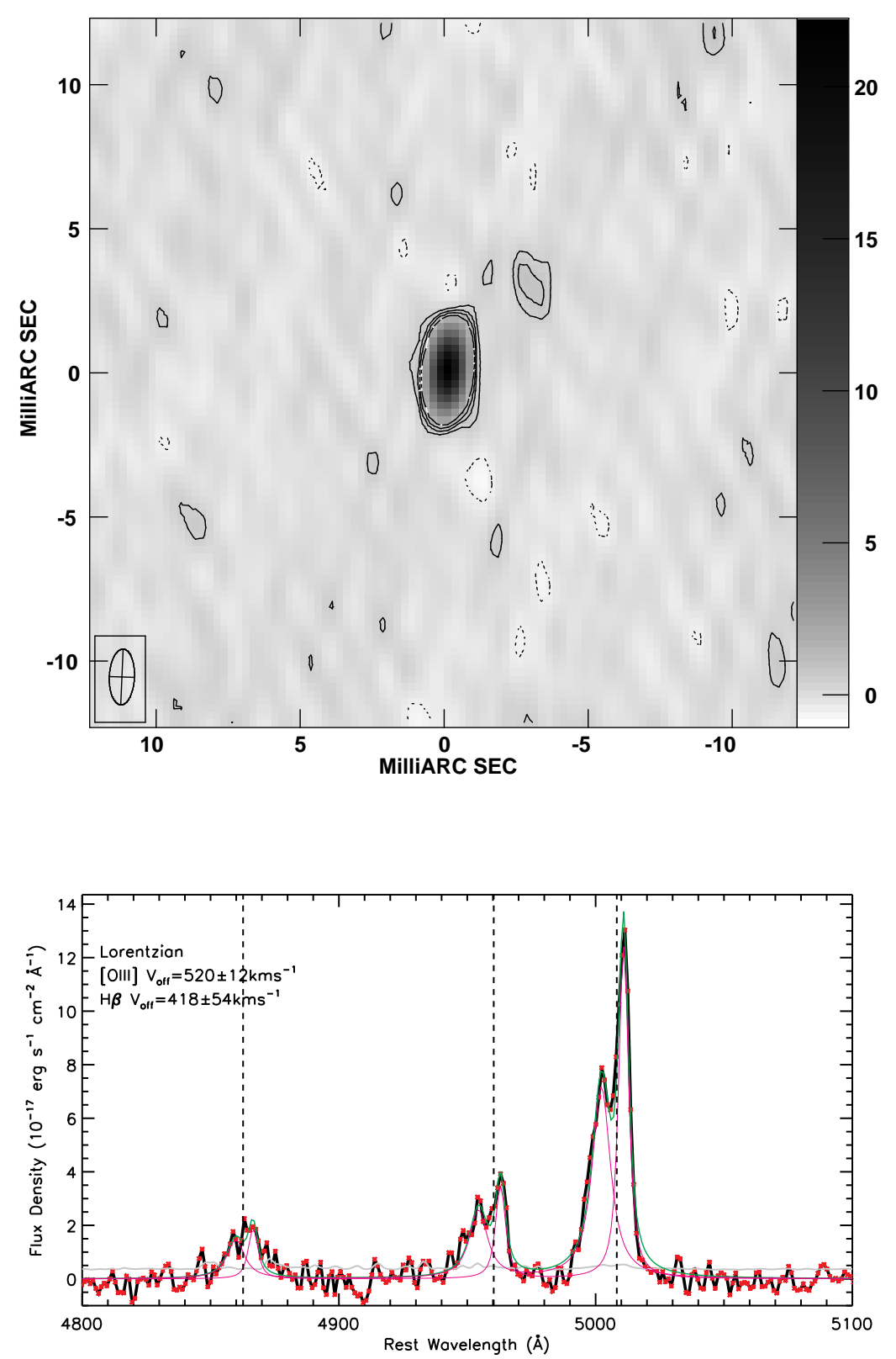

FIG. 4.- Top: VLBA 8.4 GHz image of SDSS J124358.36-005845.4. The gray scale is logarithmic, with a maximum at $22.08 \mathrm{mJy}$ beam ${ }^{-1}$. The beam is 1.94 mas $\times 0.87$ mas (corresponding to $11 \mathrm{pc} \times 4.7 \mathrm{pc}$ at the redshift of the galaxy $z=0.4092$ ), and the noise level is 0.16 mJy beam ${ }^{-1}$. Contours are given with the levels set to be $-3,3,5,7$, and 10 times the noise level in the image. Bottom: SDSS spectrum (subtracted for host-galaxy stellar continuum) along with our best spectral fits for the $\mathrm{H} \beta$ - $[\mathrm{O}$ iII $]$ region. Figure captions and symbols are the same as those in the bottom panel of Figure 2

2011) at $0 . " 6$ resolution and by HST ACS/F606W imaging at $0 . " 1$ resolution (Fu et al. 2012). However, there is tentative evidence for tidal disturbance, both from the SDSS images and the $K_{s}$-band images presented by Shen et al. (2011). There is an apparent companion $\sim 4^{\prime \prime}$ away to the southwest at $\mathrm{PA}=73^{\circ}$, which does not contribute to the [O III] emission observed in the SDSS fiber spectrum. Shen et al. (2011) presented a slit spectrum from the Apache Point Observatory 3.5m Dual Imaging Spectrograph at $\mathrm{PA}=73^{\circ}$, which suggested that the two velocity components in [O III] were spatially unresolved at $2^{\prime \prime}$ resolution.

The FIRST image shows this galaxy to be dominated by a compact component, with what may be a faint jet extending to the southeast. Figure [6 shows our VLBA image to consist of a single component, which is either unresolved or just marginally resolved. The orientation of the milliarcsecond-scale structure may appear consistent with that in the FIRST image, but this orientation is essentially the same as the CLEAN beam (which is an elliptical gaussian with a position angle of $-9^{\circ}$, east of north). 

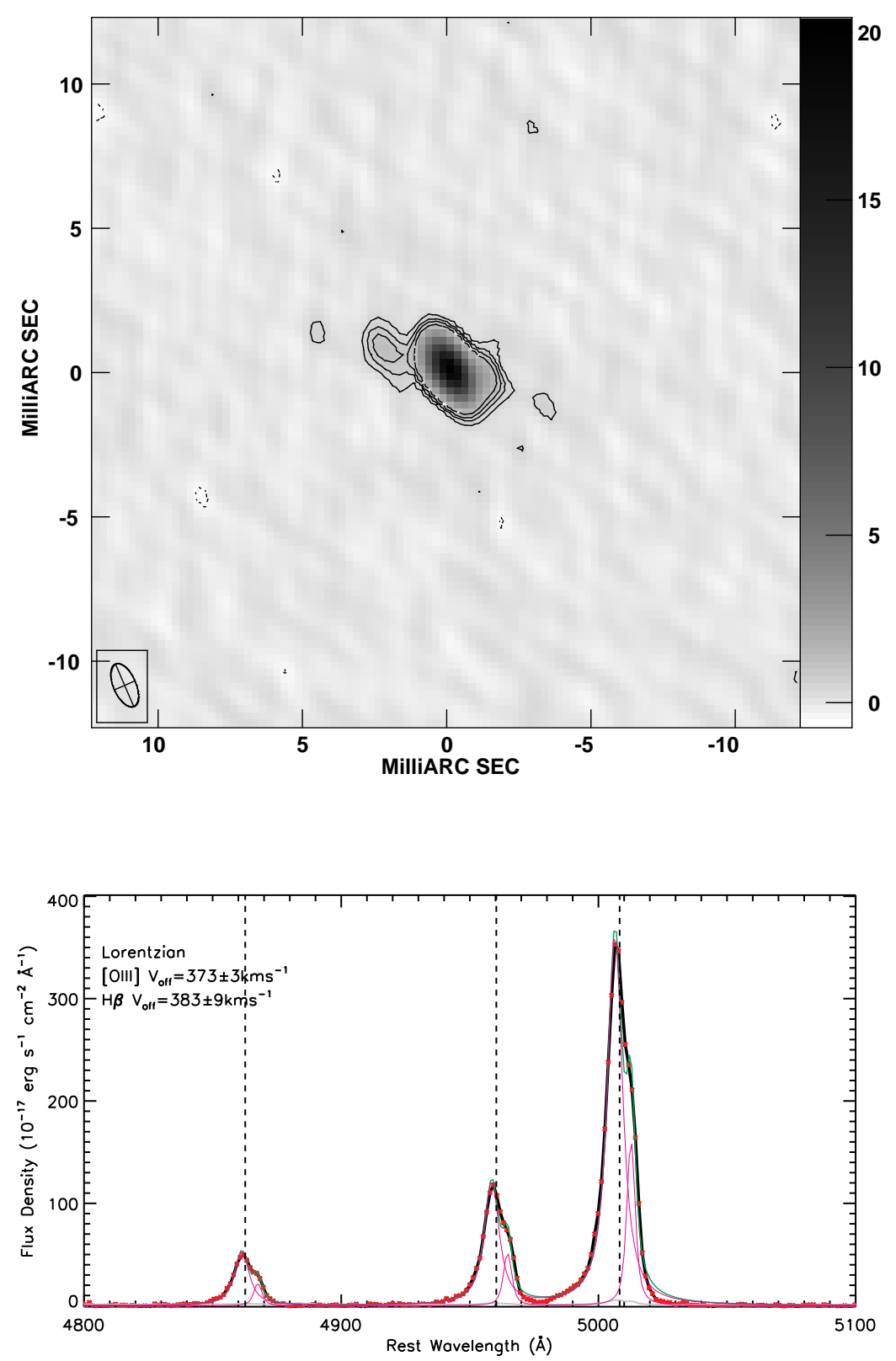

FIG. 5.- Top: VLBA $8.4 \mathrm{GHz}$ image of SDSS J135251.22+654113.2. The gray scale is logarithmic, with a maximum at $20.29 \mathrm{mJy}$ beam ${ }^{-1}$. The beam is 1.56 mas $\times 0.78$ mas (corresponding to $5.3 \mathrm{pc} \times 2.6 \mathrm{pc}$ at the redshift of the galaxy $z=0.2064$ ), and the noise level is 0.14 mJy beam ${ }^{-1}$. Contours are given with the levels set to be $-3,3,5,7$, and 10 times the noise level in the image. Bottom: SDSS spectrum (subtracted for host-galaxy stellar continuum) along with our best spectral fits for the $\mathrm{H} \beta$ - $[\mathrm{O}$ iII $]$ region. Figure captions and symbols are the same as those in the bottom panel of Figure 2

\subsection{SDSS J233313.17+004911.8 (PKS 2330+005)}

This $z=0.1699$ AGN shows double-peaked [O III] $\lambda \lambda 4959,5007$ lines in its SDSS fiber spectrum (Figure 7), with both peaks blueshifted from the systemic velocity by $480 \mathrm{~km} \mathrm{~s}^{-1}$ and $36 \mathrm{~km} \mathrm{~s}^{-1}$, respectively (Liu et al. 2010b). The SDSS images show no evidence for a double stellar core, which is confirmed by $K_{s}$-band imaging with Magellan/PANIC (Shen et al. 2011) at 0."8 resolution and by $K_{p}$-band imaging with Keck II/NIRC2 LGSAO at 0."1 resolution (Fu et al. 2012). However, there is tentative evidence for tidal disturbance, both from SDSS images and the $K_{s}$-band images presented by Shen et al. (2011). There is an apparent companion $\sim 6^{\prime \prime}$ away to the southeast at $\mathrm{PA}=117^{\circ}$, which does not contribute to the [O III] emission observed in the SDSS fiber spectrum. Shen et al. (2011) presented a slit spectrum from the APO $3.5 \mathrm{~m}$ DIS at $\mathrm{PA}=117^{\circ}$, which suggested that the two velocity components in [O III] were spatially unresolved at $1 .{ }^{\prime \prime} 5$ resolution.

This galaxy contains a radio source that has been detected in surveys from $74 \mathrm{MHz}$ to $5 \mathrm{GHz}$ (Bennett et al. 1986: Wright \& Otrupcek 1990; Becker et al. 1991; 
Gregorv \& Condon 1991; White \& Becker 1992; Griffith et al. 1995; Douglas et al. 1996; Condon et al. 1998; Cohen et al. 2007). We are unaware of any previous published observations in which the radio source is resolved. Figure 7 shows our VLBA image to consist of a single component.

\section{RESULTS AND DISCUSSION}

\subsection{Detection Rate}

Of our initial sample of 13 SDSS galaxies showing double-peaked [O III] emission lines, and with either FIRST or previous VLA detections, we detected six $(46 \%)$ in our VLBA observations to a flux density of $\sim 1 \mathrm{mJy}$. Of these six, two show likely jet structures on sub-kpc scales, while the other four are unresolved. In the other seven objects without a detectable radio core, most of the radio emission is likely from larger-scale jets or lobes. These sources are all sufficiently radio luminous that the radio emission is unlikely to be dominated by star formation in the host galaxies. The lack of masscale structure could also indicate that the central engine has recently shut down, although this explanation would require fine tuning, i.e., the fuel to the central engine shuts down only so recently that we do not see the central engine but we still see some lobe emission. As shown in Figure 1, the VLBA-detected targets on average have higher integrated fluxes at $20 \mathrm{~cm}$ from FIRST than the VLBA non-detected sources. On the other hand, the [O non-detected samples are similar. In no case have we detected an unambiguous sub-kpc dual AGN.

\subsection{Implications on the Population of Sub-kpc Dual AGNs}

We now consider what our observations imply about the potential population of sub-kpc dual AGNs. The fraction of dual AGNs on $\mathrm{kpc}$ scales among doublepeaked emission-line AGN is estimated to be of order 10\% (e.g., Shen et al. 2011; Fu et al. 2012). Another $\sim 40 \%$ of objects in the Shen et al. (2011) sample are ambiguous and some of them could harbor a sub-kpc scale dual AGN that is unresolvable with optical/NIR observations. However, we detected no sub-kpc scale dual AGN out of 13 objects in our VLBA observations, which may hint at a similar fraction $(\lesssim 10 \%)$ of sub-kpc dual AGNs among these double-peaked [O III] AGN. This would strengthen our conclusion in Shen et al. (2011) that most double-peaked profiles are caused by NLR kinematics in single AGNs rather than by the orbital motion of dual AGNs.

Another possibility is that some of the galaxies that we detect do contain a dual AGN, but that the second $\mathrm{SMBH}$ in the system is either radio faint or is of sufficiently low mass as to be undetectable in our radio observations. Accreting black holes display a "fundamental plane" relationship between their X-ray luminosity, radio luminosity, and mass (Merloni et al. 2003; Falcke et al. 2004; Gültekin et al. 2009), which allows us to estimate a $\mathrm{BH}$ mass given measurements in radio and X-rays. Accordingly, we have searched the Chandra X-ray archives for available observations of these galaxies. Only SDSS J091201.68+532036.6 (3.1) and SDSS J110851.04+065901.4 (Liu et al. 2013) have been
TABLE 3

Radio and X-Ray Luminosities, and Estimates on Black Hole MASSES

\begin{tabular}{lccc}
\hline \hline \multicolumn{1}{c}{$\begin{array}{c}\text { SDSS Name } \\
(1)\end{array}$} & $\begin{array}{c}L_{R} \\
\left.\mathrm{erg} \mathrm{s}^{-1}\right) \\
(2)\end{array}$ & $\begin{array}{c}L_{X} \\
\left.\mathrm{erg} \mathrm{s}^{-1}\right) \\
(3)\end{array}$ & $\begin{array}{c}M_{\mathrm{BH}} \\
\left(10^{8} M_{\odot}\right. \\
(4)\end{array}$ \\
\hline J091201.68+532036.6 & 34.0 & $8.3_{-1.7}^{+1.9}$ & $5.1 \pm 0.3$ \\
& $<23.8$ & $8.3_{-1.7}^{+1.9}$ & $<4.3$ \\
J113721.36+612001.2 & 10.92 & $<4.02$ & $<3.5$ \\
J124358.36-005845.4 & 225.12 & $<101$ & $<6.9$ \\
J135251.22+654113.2 & 34.44 & $<9.05$ & $<5.0$ \\
J231051.95-090011.9 & 9.24 & $<6.26$ & $<2.9$ \\
J233313.17+004911.8 & 45.36 & $<25.5$ & $<4.4$ \\
& & & \\
J000911.58-003654.7 & $<6.47$ & $<3.52$ & $<2.8$ \\
J073849.75+315611.9 & $<118$ & $<73.7$ & $<5.4$ \\
J080337.32+392633.1 & $<4.12$ & $<3.25$ & $<2.3$ \\
J085841.76+104122.1 & $<23.5$ & $<19.2$ & $<3.5$ \\
J110851.04+065901.4 & $<45.4$ & $<22.7$ & $<4.6$ \\
J135646.11+102609.1 & $<18.5$ & $<12.0$ & $<3.5$ \\
& & &
\end{tabular}

Note. - Galaxies are divided into two sets according to whether they are detected or not by VLBA. The top set corresponds to galaxies detected in our VLBA observations (see also Table 1), the lower set to the undetected galaxies (see also Table 21). Column (1): SDSS designation with J2000 coordinates. Column (2): Radio luminosity or 3- $\sigma$ upper limit. Column (3): X-ray luminosity or 3- $\sigma$ upper limit. Column (4): Estimated black hole mass or upper limit assuming the fundamental plane relationship between X-ray luminosity, radio luminosity and black hole mass (Gültekin et al. 2009).

observed and detected previously in targeted X-ray observations. For the remaining galaxies, we examined the ROSAT All-Sky Survey 13 for a possible X-ray counterpart. In no case, do we detect a ROSAT X-ray source at the location of these galaxies. We converted the ROSAT X-ray flux limits to rest-frame $2-10 \mathrm{keV}$ flux limits using the online PIMMS too 14 .

Table 3 summarizes what the combined radio and $\mathrm{X}$ ray measurements imply about the masses, or upper limits on the masses, of SMBHs in the nuclei of these galaxies. For the galaxy with both radio and X-ray detections (SDSS J091201.68+532036.6), we estimate the masses of a potential dual SMBH system (one being radio bright and one being radio faint, i.e., below our detection limit) making the following assumptions. For the radio factor in the fundamental plane relation, we use either the flux density of the compact component or the $3 \sigma$ upper limit. For the X-ray factor in the fundamental plane relation, we assume that the two putative SMBHs would contribute equally to the X-ray flux. While this approach clearly does not yield a unique solution, it is indicative of the characteristics of a dual SMBH system, if the second black hole is not radio bright.

For the remaining galaxies, we use the $(3 \sigma)$ upper limit in the radio image and that on the $\mathrm{X}$-ray flux to constrain the mass of an SMBH in the galaxy 15 . This approach is also clearly not unique, but it should suffice to provide an estimate of the possible SMBH masses.

Typical $3 \sigma$ upper limits on the mass of a second $\mathrm{SMBH}$ in the nuclei of these galaxies are $(3-7) \times 10^{8}$

\footnotetext{
13 http://www.xray.mpe.mpg.de/cgi-bin/rosat/data-browser

14 http://cxc.harvard.edu/toolkit/pimms.jsp

15 For consistency with the other radio undetected galaxies, we also quote the upper limit on the X-ray flux for the galaxy SDSS J110851.04+065901.4, which is detected by Chandra.
} 

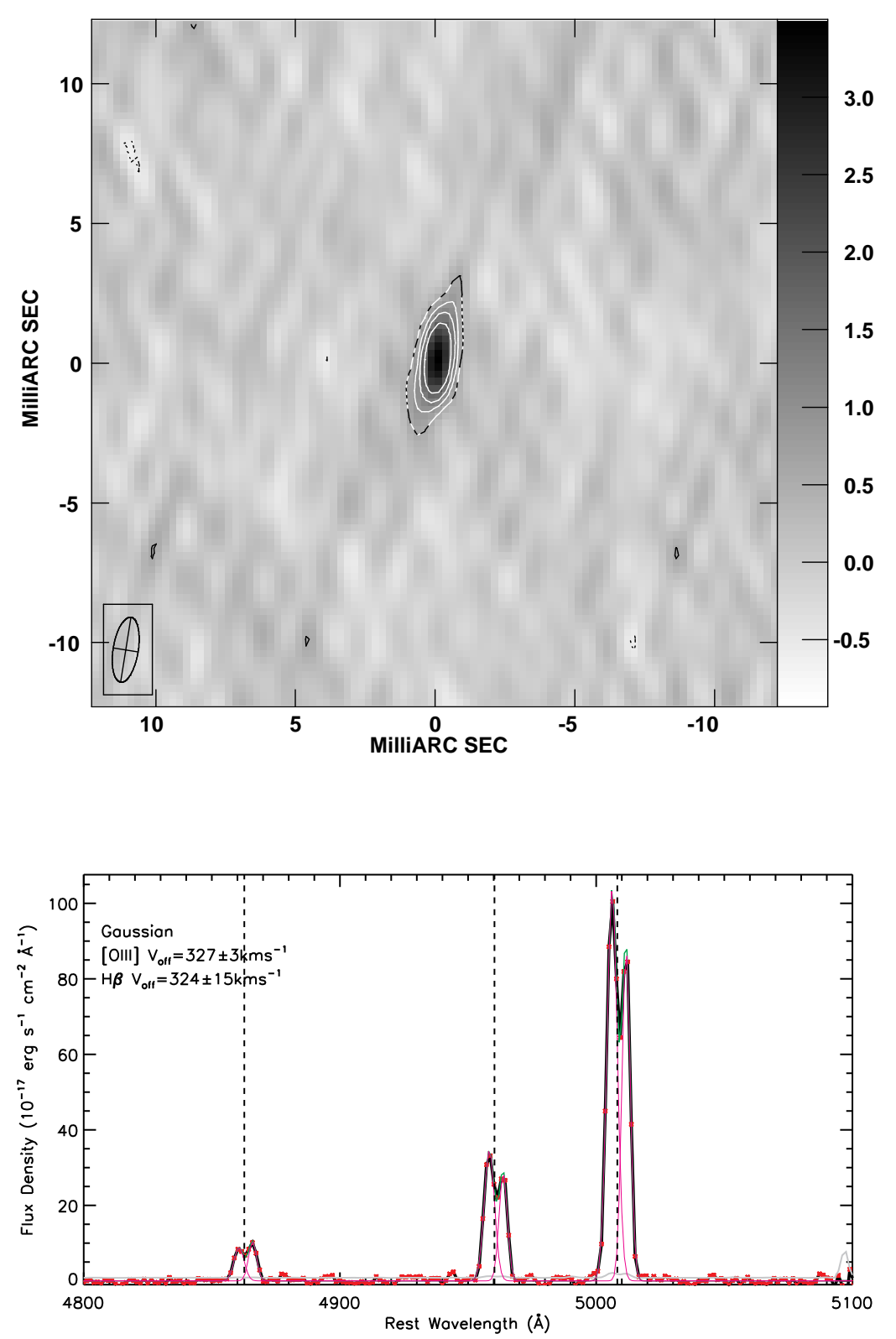

Fig. 6. - Top: VLBA $8.4 \mathrm{GHz}$ image of SDSS J231051.95-090011.9. The gray scale is linear over the range -0.90 to $3.47 \mathrm{mJy}$ beam ${ }^{-1}$. The beam is 2.35 mas $\times 0.90$ mas (corresponding to $4.1 \mathrm{pc} \times 1.6 \mathrm{pc}$ at the redshift of the galaxy $z=0.0944$ ), and the noise level is 0.18 mJy beam ${ }^{-1}$. Contours are given with the levels set to be $-3,3,5,7$, and 10 times the noise level in the image. Bottom: SDSS spectrum (subtracted for host-galaxy stellar continuum) along with our best spectral fits for the $\mathrm{H} \beta$-[O III] region. Figure captions and symbols are the same as those in the bottom panel of Figure 2

$M_{\odot}$. We stress that these black hole mass limits should be viewed as indicative, particularly given that Gültekin et al. (2009) find that the fundamental plane relation that we have assumed has a scatter of 0.77 dex. Nonetheless, it is possible that these galaxies might still contain a second SMBH, whose presence may become apparent with significantly deeper radio and X-ray observations.

\subsection{Comparison with Previous Work and Remarks on Future Directions}

Tingav \& Wavth (2011) have conducted a similar search for dual SMBHs with VLBA. While their fraction of detected sources was lower (2 of 12 or $17 \%$ ), they also found no dual AGN candidates. Taken together, these observations are consistent with the fraction of (radio-bright) dual SMBHs on sub-kpc to pcscale separations being similar to that on larger separations, namely $0.1 \%$, though the sample remains small. As nearly half of the objects in the Liu et al. (2010b) sample have a radio counterpart and that new samples of AGNs with double-peaked narrow emission lines are available (e.g., Lvu \& Liu 2016; Yuan et al. 2016), there are ample possibilities for expanding the set of double- 


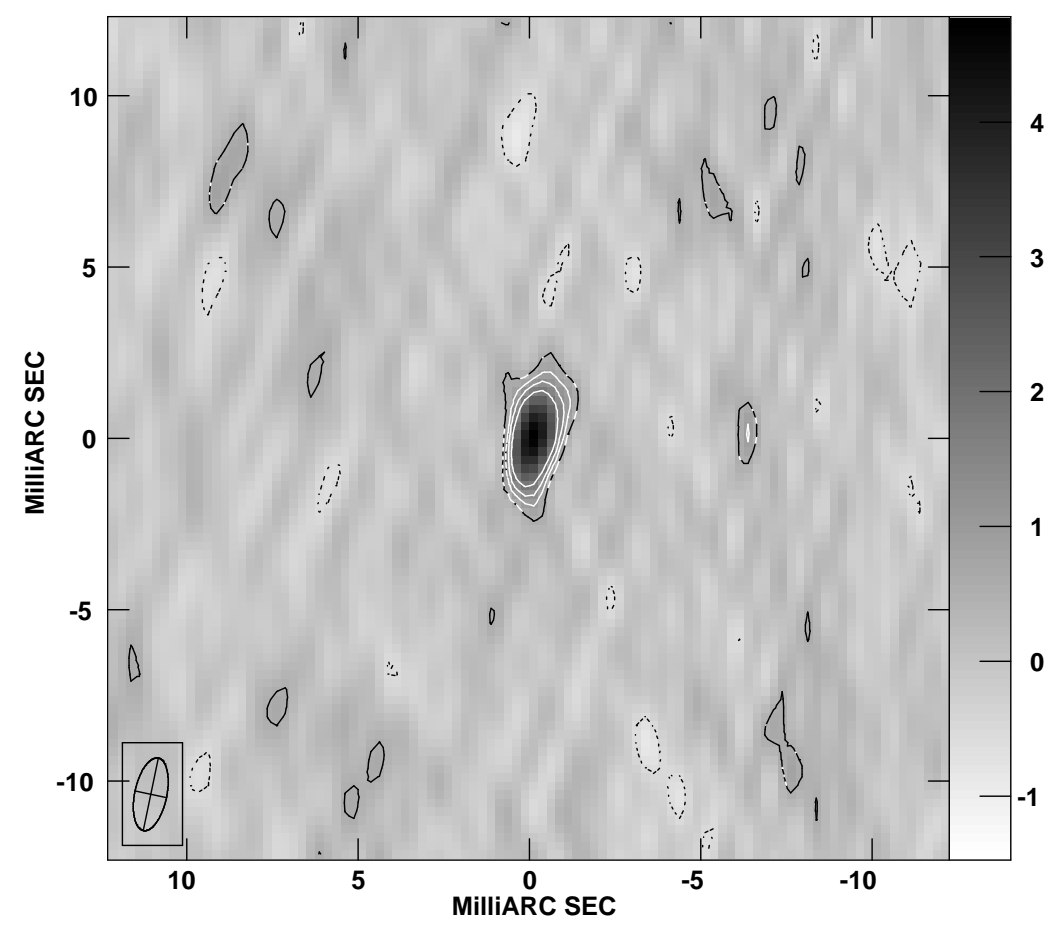

4

3

2

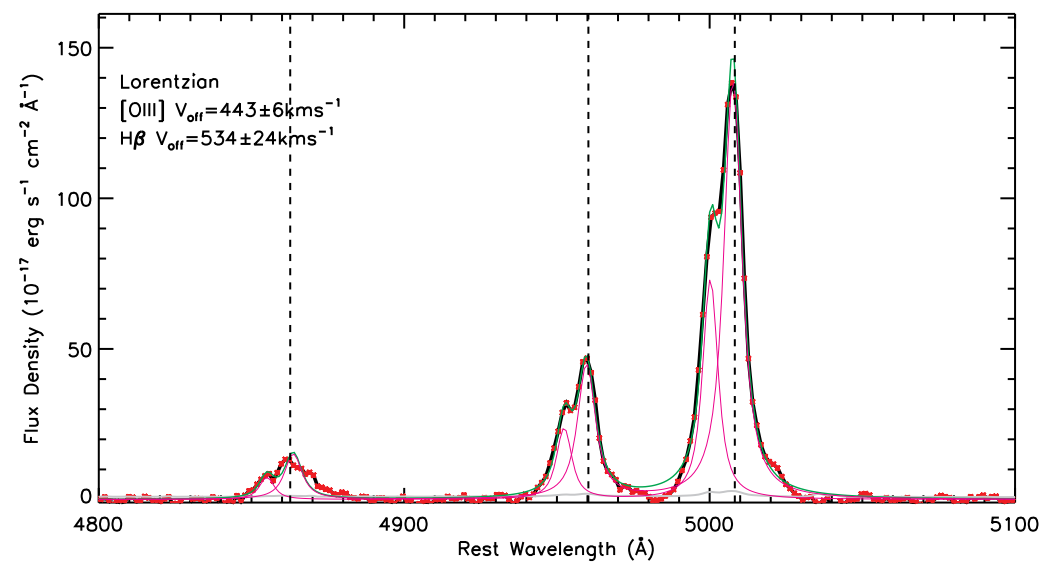

Fig. 7. - Top: VLBA $8.4 \mathrm{GHz}$ image of SDSS J233313.17+004911.8. The gray scale is linear over the range -1.43 to $4.74 \mathrm{mJy}$ beam ${ }^{-1}$. The beam is 2.16 mas $\times 0.94$ mas (corresponding to $6.3 \mathrm{pc} \times 2.7 \mathrm{pc}$ at the redshift of the galaxy $z=0.1699$ ), and the noise level is 0.24 mJy beam ${ }^{-1}$. Contours are given with the levels set to be $-3,3,5,7$, and 10 times the noise level in the image. Bottom: SDSS spectrum (subtracted for host-galaxy stellar continuum) along with our best spectral fits for the $\mathrm{H} \beta$ - $[\mathrm{O}$ iII $]$ region. Figure captions and symbols are the same as those in the bottom panel of Figure 2

peaked line emission galaxies that have been examined for possible dual AGNs on sub-kpc scales.

Having a higher sensitivity than VLBA on larger scales, VLA is a better match in terms of searching for kpc-scale dual AGNs (e.g., Burke-Spolaor et al. 2014; Fu et al. 2015; Müller-Sánchez et al. 2015), kpcscale jet-cloud interactions, or kpc-separation compact radio sources. Nevertheless, VLBA is superior in terms of spatial resolution, and has the potential to resolve subkpc projected pairs, and to test the probability of subkpc jets as the origin for double-peaked narrow-line pro- files, where the NLR emission is unresolved on kpc scales (e.g., Wrobel et al. 2014). It would also be interesting to carry out radio follow ups for new large samples of AGNs with double-peaked narrow emission lines at higher redshift (e.g., Lvu \& Liu 2016; Yuan et al. 2016) to address their possible redshift and/or luminosity evolution (e.g., Yu et al. 2011).

We thank S. Burke-Spolaor for helpful discussions and the anonymous referee for a careful and useful report that improved the paper. Y.S. acknowledges support from the 
Alfred P. Sloan Foundation and NSF grant 1715579.

The NANOGrav project receives support from National Science Foundation Physics Frontier Center award number 1430284. The Long Baseline Observatory is a facility of the National Science Foundation operated under cooperative agreement by Associated Universities, Inc. This research has made use of the NASA/IPAC Extragalactic Database (NED) which is operated by the Jet Propulsion Laboratory, California Institute of Technology, under contract with the National Aeronautics and Space Administration. This research has made use of NASA's Astrophysics Data System. This research has made use of data obtained from the High Energy Astrophysics Science Archive Research Center (HEASARC), provided by NASA's Goddard Space Flight Center. This research has made use of data obtained from the Chandra Source Catalog, provided by the Chandra X-ray Center (CXC) as part of the Chandra Data Archive. Part of this research was carried out at the Jet Propulsion Laboratory, California Institute of Technology, under a contract with the National Aeronautics and Space Administration.

Funding for the SDSS and SDSS-II has been provided by the Alfred P. Sloan Foundation, the Participating Institutions, the National Science Foundation, the U.S. Department of Energy, the National Aeronautics and Space Administration, the Japanese Monbukagakusho, the Max Planck Society, and the Higher Education Funding Council for England. The SDSS website is http://www.sdss.org/.

The SDSS is managed by the Astrophysical Research Consortium for the Participating Institutions. The Participating Institutions are the American Museum of Natural History, Astrophysical Institute Potsdam, University of Basel, University of Cambridge, Case Western Reserve University, University of Chicago, Drexel University, Fermilab, the Institute for Advanced Study, the Japan Participation Group, Johns Hopkins University, the Joint Institute for Nuclear Astrophysics, the Kavli Institute for Particle Astrophysics and Cosmology, the Korean Scientist Group, the Chinese Academy of Sciences (LAMOST), Los Alamos National Laboratory, the Max Planck-Institute for Astronomy (MPIA), the Max Planck Institute for Astrophysics (MPA), New Mexico State University, Ohio State University, University of Pittsburgh, University of Portsmouth, Princeton University, the United States Naval Observatory, and the University of Washington.

Facilities: VLBA, Sloan

\section{REFERENCES}

Abazajian, K. N., Adelman-McCarthy, J. K., Agüeros, M. A., et al. 2009, ApJS, 182, 543

Amaro-Seoane, P., Aoudia, S., Babak, S., et al. 2013, GW Notes, 6,4

An, T., Paragi, Z., Frey, S., et al. 2013, MNRAS, 433, 1161

Arzoumanian, Z., Brazier, A., Burke-Spolaor, S., et al. 2016, ApJ, 821,13

Audley, H., Babak, S., Baker, J., et al. 2017, ArXiv e-prints 1702.00786, arXiv:1702.00786

Axon, D. J., Marconi, A., Capetti, A., et al. 1998, ApJ, 496, L75

Babak, S., Petiteau, A., Sesana, A., et al. 2016, MNRAS, 455, 1665

Barrows, R. S., Sandberg Lacy, C. H., Kennefick, J., et al. 2013, ApJ, 769, 95

Barrows, R. S., Stern, D., Madsen, K., et al. 2012, ApJ, 744, 7

Becker, R. H., White, R. L., \& Edwards, A. L. 1991, ApJS, 75, 1

Becker, R. H., White, R. L., \& Helfand, D. J. 1995, ApJ, 450, 559

Begelman, M. C., Blandford, R. D., \& Rees, M. J. 1980, Nature, 287, 307

Bennett, C. L., Lawrence, C. R., Burke, B. F., Hewitt, J. N., \& Mahoney, J. 1986, ApJS, 61, 1

Berczik, P., Merritt, D., Spurzem, R., \& Bischof, H.-P. 2006, ApJ, 642, L21

Bianchi, S., Chiaberge, M., Piconcelli, E., Guainazzi, M., \& Matt, G. 2008, MNRAS, 386, 105

Boroson, T. A., \& Lauer, T. R. 2009, Nature, 458, 53

Burke-Spolaor, S., Brazier, A., Chatterjee, S., et al. 2014, ArXiv e-prints 1402.0548, arXiv:1402.0548

Charisi, M., Bartos, I., Haiman, Z., et al. 2016, MNRAS, 463, 2145

Chornock, R., Bloom, J. S., Cenko, S. B., et al. 2010, ApJ, 709, L39

Cohen, A. S., Lane, W. M., Cotton, W. D., et al. 2007, AJ, 134, 1245

Comerford, J. M., Gerke, B. F., Stern, D., et al. 2012, ApJ, 753, 42

Comerford, J. M., Pooley, D., Gerke, B. F., \& Madejski, G. M. 2011, ApJ, 737, L19

Comerford, J. M., Schluns, K., Greene, J. E., \& Cool, R. J. 2013, ApJ, 777, 64

Comerford, J. M., Gerke, B. F., Newman, J. A., et al. 2009, ApJ, 698,956

Condon, J. J., Cotton, W. D., Greisen, E. W., et al. 1998, AJ, 115,1693
Crenshaw, D. M., Schmitt, H. R., Kraemer, S. B., Mushotzky, R. F., \& Dunn, J. P. 2010, ApJ, 708, 419

D’Orazio, D. J., Haiman, Z., Duffell, P., MacFadyen, A., \& Farris, B. 2016, MNRAS, 459, 2379

D'Orazio, D. J., Haiman, Z., \& Schiminovich, D. 2015, Nature, 525,351

Douglas, J. N., Bash, F. N., Bozyan, F. A., Torrence, G. W., \& Wolfe, C. 1996, AJ, 111, 1945

Eracleous, M., Boroson, T. A., Halpern, J. P., \& Liu, J. 2012, ApJS, 201, 23

Evans, I. N., Primini, F. A., Glotfelty, K. J., et al. 2010, ApJS, 189,37

Falcke, H., Körding, E., \& Markoff, S. 2004, A\&A, 414, 895

Ferrarese, L., \& Ford, H. 2005, Space Sci. Rev., 116, 523

Filho, M. E., Barthel, P. D., \& Ho, L. C. 2006, A\&A, 451, 71

Fischer, T. C., Crenshaw, D. M., Kraemer, S. B., et al. 2011, ApJ, 727, 71

Fu, H., Myers, A. D., Djorgovski, S. G., et al. 2015, ApJ, 799, 72

Fu, H., Yan, L., Myers, A. D., et al. 2012, ApJ, 745, 67

Fu, H., Zhang, Z.-Y., Assef, R. J., et al. 2011, ApJ, 740, L44

Ge, J.-Q., Hu, C., Wang, J.-M., Bai, J.-M., \& Zhang, S. 2012, ApJS, 201, 31

Gerke, B. F., Newman, J. A., Lotz, J., et al. 2007, ApJ, 660, L23

Gower, J. F. R., Scott, P. F., \& Wills, D. 1967, MmRAS, 71, 49

Graham, M. J., Djorgovski, S. G., Stern, D., et al. 2015a, Nature, 518,74

-. 2015b, MNRAS, 453, 1562

Greene, J. E., Zakamska, N. L., Ho, L. C., \& Barth, A. J. 2011, ApJ, 732, 9

Gregory, P. C., \& Condon, J. J. 1991, ApJS, 75, 1011

Griffith, M. R., Wright, A. E., Burke, B. F., \& Ekers, R. D. 1995, ApJS, 97, 347

Gültekin, K., Cackett, E. M., Miller, J. M., et al. 2009, ApJ, 706, 404

Hales, S. E. G., Masson, C. R., Warner, P. J., \& Baldwin, J. E. 1990, MNRAS, 246, 256

Hales, S. E. G., Waldram, E. M., Rees, N., \& Warner, P. J. 1995, MNRAS, 274, 447

Hennawi, J. F., Strauss, M. A., Oguri, M., et al. 2006, AJ, 131, 1

Hennawi, J. F., Myers, A. D., Shen, Y., et al. 2010, ApJ, 719, 1672

Hobbs, G., Archibald, A., Arzoumanian, Z., et al. 2010, Classical and Quantum Gravity, 27, 084013 
Hopkins, P. F., Hernquist, L., Cox, T. J., \& Kereš, D. 2008, ApJS, 175, 356

Ju, W., Greene, J. E., Rafikov, R. R., Bickerton, S. J., \& Badenes, C. 2013, ApJ, 777, 44

Junkkarinen, V., Shields, G. A., Beaver, E. A., et al. 2001, ApJ, 549, L155

Kauffmann, G., \& Haehnelt, M. 2000, MNRAS, 311, 576

Khan, F. M., Just, A., \& Merritt, D. 2011, ApJ, 732, 89

Kharb, P., Lal, D. V., \& Merritt, D. 2017, Nature Astronomy, 1, 727

Komossa, S., Burwitz, V., Hasinger, G., et al. 2003, ApJ, 582, L15

Kormendy, J., \& Bender, R. 2009, ApJ, 691, L142

Kormendy, J., \& Ho, L. C. 2013, ARA\&A, 51, 511

Kormendy, J., \& Richstone, D. 1995, ARA\&A, 33, 581

Koss, M., Mushotzky, R., Treister, E., et al. 2011, ApJ, 735, L42

Kudryavtseva, N. A., Britzen, S., Witzel, A., et al. 2011, A\&A, 526, A 51

Lara, L., Cotton, W. D., Feretti, L., et al. 2001, A\&A, 370, 409

Liu, F. K. 2004, MNRAS, 347, 1357

Liu, T., Gezari, S., Heinis, S., et al. 2015, ApJ, 803, L16

Liu, X., Civano, F., Shen, Y., et al. 2013, ApJ, 762, 110

Liu, X., Greene, J. E., Shen, Y., \& Strauss, M. A. 2010a, ApJ, 715, L30

Liu, X., Guo, H., Shen, Y., Greene, J. E., \& Strauss, M. A. 2017, ArXiv e-prints 1712.01866, arXiv:1712.01866

Liu, X., Shen, Y., Bian, F., Loeb, A., \& Tremaine, S. 2014, ApJ, 789,140

Liu, X., Shen, Y., \& Strauss, M. A. 2012, ApJ, 745, 94

Liu, X., Shen, Y., Strauss, M. A., \& Greene, J. E. 2010b, ApJ, 708,427

Liu, X., Shen, Y., Strauss, M. A., \& Hao, L. 2011, ApJ, 737, 101

Lodato, G., Nayakshin, S., King, A. R., \& Pringle, J. E. 2009, MNRAS, 398, 1392

Lyu, Y., \& Liu, X. 2016, MNRAS, 463, 24

Maness, H. L., Taylor, G. B., Zavala, R. T., Peck, A. B., \& Pollack, L. K. 2004, ApJ, 602, 123

Mayer, L., Kazantzidis, S., Madau, P., et al. 2007, Science, 316 1874

McGurk, R. C., Max, C. E., Rosario, D. J., et al. 2011, ApJ, 738, L2

Merloni, A., Heinz, S., \& di Matteo, T. 2003, MNRAS, 345, 1057

Merritt, D. 2013, Dynamics and Evolution of Galactic Nuclei, Princeton Series in Astrophysics (Princeton University Press)

Milosavljević, M., \& Merritt, D. 2001, ApJ, 563, 34

Müller-Sánchez, F., Comerford, J. M., Nevin, R., et al. 2015, ApJ, 813, 103

Myers, A. D., Richards, G. T., Brunner, R. J., et al. 2008, ApJ, 678,635

Myers, S. T., Jackson, N. J., Browne, I. W. A., et al. 2003, MNRAS, 341, 1

Napier, P. J., Bagri, D. S., Clark, B. G., et al. 1994, IEEE Proceedings, 82, 658

Nevin, R., Comerford, J., Müller-Sánchez, F., Barrows, R., \& Cooper, M. 2016, ApJ, 832, 67

Owen, F. N., O’Dea, C. P., Inoue, M., \& Eilek, J. A. 1985, ApJ, 294, L85

Oyaizu, H., Lima, M., Cunha, C. E., et al. 2008, ApJ, 674, 768
Preto, M., Berentzen, I., Berczik, P., \& Spurzem, R. 2011, ApJ, 732, L26

Quinlan, G. D. 1996, NewA, 1, 35

Rodriguez, C., Taylor, G. B., Zavala, R. T., et al. 2006, ApJ, 646, 49

Rosario, D. J., Shields, G. A., Taylor, G. B., Salviander, S., \& Smith, K. L. 2010, ApJ, 716, 131

Runnoe, J. C., Eracleous, M., Mathes, G., et al. 2015, ApJS, 221,

Runnoe, J. C., Eracleous, M., Pennell, A., et al. 2017, MNRAS, 468,1683

Schmitt, H. R., Donley, J. L., Antonucci, R. R. J., et al. 2003, ApJ, 597, 768

Sesana, A. 2010, ApJ, 719, 851

Sesana, A., Haiman, Z., Kocsis, B., \& Kelley, L. Z. 2017, ArXiv e-prints 1703.10611, arXiv:1703.10611

Shen, Y. 2009, ApJ, 704, 89

Shen, Y., Liu, X., Greene, J. E., \& Strauss, M. A. 2011, ApJ, 735, 48

Shen, Y., Liu, X., Loeb, A., \& Tremaine, S. 2013, ApJ, 775, 49

Shen, Y., Hennawi, J. F., Shankar, F., et al. 2010, ApJ, 719, 1693

Sillanpaa, A., Haarala, S., Valtonen, M. J., Sundelius, B., \&

Byrd, G. G. 1988, ApJ, 325, 628

Smith, K. L., Shields, G. A., Bonning, E. W., et al. 2010, ApJ, 716,866

Smith, K. L., Shields, G. A., Salviander, S., Stevens, A. C., \& Rosario, D. J. 2011, ArXiv e-prints 1108.0383, arXiv:1108.0383

Steinborn, L. K., Dolag, K., Comerford, J. M., et al. 2016, MNRAS, 458, 1013

Tingay, S. J., \& Wayth, R. B. 2011, AJ, 141, 174

Tsalmantza, P., Decarli, R., Dotti, M., \& Hogg, D. W. 2011, ApJ, 738,20

Valtonen, M. J., Lehto, H. J., Nilsson, K., et al. 2008, Nature, 452,851

Valtonen, M. J., Zola, S., Ciprini, S., et al. 2016, ApJ, 819, L37

Vaughan, S., Uttley, P., Markowitz, A. G., et al. 2016, MNRAS, 461,3145

Volonteri, M., Haardt, F., \& Madau, P. 2003, ApJ, 582, 559

Wang, J., Chen, Y., Hu, C., et al. 2009, ApJ, 705, L76

Wang, L., Greene, J. E., Ju, W., et al. 2017, ApJ, 834, 129

White, R. L., \& Becker, R. H. 1992, ApJS, 79, 331

Williams, D. R. A., McHardy, I. M., Baldi, R. D., et al. 2017, MNRAS, 472,3842

Wright, A., \& Otrupcek, R. 1990, in PKS Catalog (1990)

Wrobel, J. M., Comerford, J. M., \& Middelberg, E. 2014, ApJ, 782,116

Wyithe, J. S. B., \& Loeb, A. 2003, ApJ, 595, 614

Xu, D., \& Komossa, S. 2009, ApJ, 705, L20

York, D. G., Adelman, J., Anderson, Jr., J. E., et al. 2000, AJ, 120,1579

Yu, Q. 2002, MNRAS, 331, 935

Yu, Q., Lu, Y., Mohayaee, R., \& Colin, J. 2011, ApJ, 738, 92

Yuan, S., Strauss, M. A., \& Zakamska, N. L. 2016, MNRAS, 462, 1603

Zheng, Z.-Y., Butler, N. R., Shen, Y., et al. 2016, ApJ, 827, 56

Zhou, H., Wang, T., Zhang, X., Dong, X., \& Li, C. 2004, ApJ, 604, L33 